\title{
VON SITTEN UND BRÄUCHEN IM MZAB
}

\author{
KARL SUTER
}

Eigenartigerweise sind gewisse bei uns vorkommende Sitten und Bräuche, wie Flursegnungen, Bittgänge, Gedenkfeiern, Frontage, Jungmännerbünde auch beim Berberstamm der Mozabiten, der die Landschaft des Mzab (algerische Sahara) bewohnt, verbreitet. Diese Berber, die in religiöser Hinsicht Mohammedaner, und zwar Ibaditen, sind, gründeten dort die folgenden sieben Ksur (Einzahl Ksar, Name für befestigte Siedlung) mitsamt ihren Palmgärten: Ghardaia, Melika, Ben Isguen, Bou Noura, El Ateuf, Guerrara, Berrian. ${ }^{1}$

Jede dieser Siedlungen führt, wie gewisse von Katholiken bewohnte Dörfer unseres Alpenraumes, alljährlich im März² eine Flursegnung durch, zu einer Zeit also, da im Palmgarten das Getreide (Weizen, Gerste) der Reife entgegengeht und die Dattelpalmen bestäubt werden. In einer Prozession, die vom Scheich, dem Vorsteher der ibaditischen Moschee und des geistlichen Kollegiums (Halga), angeführt wird, durchziehen Jünglinge und Männer - in Ghardaia etwa 200 bis 300 Mann - den ganzen Palmenhain, die marschtüchtigen zu Fuß, die andern auf Eseln. Während des Rundganges, der am Vormittag bei der Moschee beginnt und je nach der Größe der Oase zwei bis vier Stunden dauert, werden Suren aus dem Koran rezitiert und Allah um seinen Segen für den Garten angefleht.

Sie suchen auch benachbarte, längst zerfallene Siedlungen auf, wie auch die Gräber bedeutender Scheiche, bei welcher Gelegenheit die Korangelehrten (Azzaba) Wissenswertes aus deren Leben erzählen und im besondern an deren Wohltaten erinnern. Unterwegs pflegen die Prozessionsteilnehmer wiederholt zu rasten, Brot und Datteln zu essen und an die Armen zu verteilen. In Ghardaia ist es üblich, die Prozession am Wadi nahe der Talsperre, welche zum Friedhof Ammi Said führt, bei Gebet, Speise und Trank (Datteln, Butter, Milch) zu beendigen. Eine weitere Zusammenkunft findet am Wadi noch am Spätnachmittag statt, wobei von vielen Haushaltungen gespendeter Kuskus an die Armen verteilt wird.

Die Flursegnung wird immer auf einen Montag angesetzt. Auf welchen, das gibt der Scheich der betreffenden Siedlung zeitig in der Moschee bekannt. Der Montag wird gewählt, weil, wie mir die Leute sagten, an diesem Wochentag der Prophet Mohammed geboren worden sei.

In Zeiten großer Trockenheit, wenn die Brunnen des Palmgartens fast kein Wasser mehr liefern, werden Bittgänge um Regen veranstaltet. In der Morgenfrühe des vom Scheich vorgesehenen Tages suchen, wie Leutnant Charlet $^{3}$ (S. 41) berichtet, die Männer mit den Korangelehrten an ihrer Spitze den Wadi und die verschiedenen Tobel, die im Gebiete des Palmgartens in ihn einmünden, auf und flehen dabei um Regen. Den Abschluß des Bittganges bildet ein Schlachtopfer. An einer bestimmten Stelle des Gartens werden die Kamele, welche von der mozabitischen Bevölkerung des betreffenden Ksar gekauft wurden, getötet, das Fleisch in großen Kesseln gekocht und hierauf Stücke davon, oft mit Kuskus zusammen, unter alle Bittgänger, insbesondere die Armen, verteilt. Die Mozabiten glauben auf diese Weise, wie A.-M. Goichon ${ }^{4}$ (Band II, S. 43) schreibt, der Barmherzigkeit und Güte Allahs teilhaftig zu werden und Regen zu erwirken.

Im Mzab werden noch andere im Dienst der Religion stehende und mit allgemeinen

1 Die Ortsnamen werden in französischer Schreibweise wiedergegeben, nicht aber die arabischen Eigennamen und Begriffe.

2 Berrian immer am ersten Montag im April.

3 Leutnant Charlet: Les palmiers du Mzab. Bulletin de la Société de Géographie d'Alger, Alger 1905.

4 A.-M. Goichon: La vie féminine au Mzab, Band I, Paris 1927; Band II, Paris 1931. 
Speisungen verbundene Zusammenkünfte durchgeführt, immer im Beisein des geistlilichen Kollegiums. Sie heißen Maruf. Besonders bekannt ist zum Beispiel der Maruf, den Melika jedes Winterhalbjahr zu Ehren seines bedeutendsten Scheichs, des Sidi Aissa, abhält. Seine Nachkommen sammeln unter den Mozabiten des Ksar, ja selbst den Ortsabwesenden, Geld für den Ankauf von mehreren, oft 10 bis 15 Kamelen. In der Morgenfrühe eines bestimmten Tages, stets vor Sonnenaufgang, werden diese Kamele ritusgemäß geschlachtet und deren Fleisch, den fünf Quartieren der Siedlung entsprechend, zu fünf Portionen aufgehäuft. Jede Portion entspricht in ihrem Ausmaß der Bevölkerungszahl des Quartiers. Sie wird weiter unterteilt, und zwar in so viele Stücke, als das Quartier Einwohner zählt; Säuglinge und Ortsabwesende werden dabei mitberücksichtigt. Hierauf gehen Männer von $\mathrm{H}$ aus zu Haus, um die den einzelnen Familien zukommende Anzahl von Fleischstücken abzuliefern. Im ganzen gelangen jedesmal rund 3000 Stück zur Verteilung. Die übriggebliebenen Teile der Tiere, wie Kopf, Beine, Haut, Fett und Eingeweide, werden im Laufe des Vormittags auf dem Marktplatz versteigert. Das Geld, das dabei eingeht, dient zur Deckung eines Teiles der Unkosten. Die von Gebeten begleitete Feier, die gewöhnlich auf einen Montag, ausnahmsweise auf einen Donnerstag angesetzt wird, ist unter dem Name Djemel Sidi Aissa (die Kamele des Sidi Aissa) bekannt. Welch ein Motiv ihr zugrunde liegt, vermochte ich nicht herauszubringen. Wie mir aber scheint, handelt es sich um eine Maßnahme zur Bekämpfung von Hunger und Not unter der eigenen Bevölkerung.

Einen ähnlichen Verlauf nimmt der alle drei bis fünf Jahre zu Ehren des Scheichs Sidi Brahim stattfindende Maruf in El Ateuf. Bei diesem Anla $\beta$ müssen ein bis zwei Dutzend gemeinsam gekaufter Kamele auf dessen Friedhof ihr Leben lassen. Ihr Fleisch wird an Ort und Stelle gekocht und bildet dann mit Brot zusammen, das wohlhabende Familien spenden, eine wahre Gaumenfreude. Wer immer sich gerade in ElAteuf aufhält, ist zum Mittun eingeladen. Bei diesem Maruf sind stets 1500 bis 2000 Personen zu bewirten.

Zusammenkünfte verwandter Art führt auch das im Mzab gelegene Araberdorf Metlili, das dem Nomadenstamm der Schamba gehört, durch. In der Zeit von Oktober bis Dezember, wenn die meisten Stammesangehörigen im Gebiete weilen, suchen die einzelnen Sippen die Grabkapelle ihres Heiligen (Marabut) auf, so zum Beispiel die Sippe der Schorfa die Grabkapelle des Sidi Mulay Sliman oder die Sippe der Beni Merzug jene des Sidi Hadj Mussa. Diese Kapellen erheben sich meist auf Hügelkuppen der nächsten Umgebung. Und auch in dieser Oase: Geldsammlung unter den Sippengliedern für den Ankauf von Tieren (Kamele, Schafe), Schlachten der Tiere bei der Grabkapelle und Verteilen des gekochten Fleisches.

Diese Flursegnungen und Prozessionen sind den Mozabiten heilig. Sie führen sie selbst unter erschwerten Umständen durch, wie in Kriegszeiten, wenn das Fleisch rationiert ist. Doch wie seltsam! Monatelang war während des $Z$ weiten Weltkrieges fast kein Fleisch zu sehen - zur Abhaltung ihrer Feiern tauchten aber Schlachttiere in genügend großer Zahl auf.

Verschiedene Ksur veranstalten jährlich Erinnerungsfeiern an geschichtliche Ereignisse, so die Bevölkerung von Ben Isguen im Mai und August, um an den Untergang ihres alten Ksar Mourki zu erinnern. Sie sucht die Ruinenstätte auf, verrichtet auf dem Platz, wo einst die Moschee stand, die Gebete und läßt Fleisch mitgeführter und an Ort und Stelle getöteter Schafe, ferner Datteln, Brot und kleinere Geldbeträge unter die Armen verteilen. Die Leute von Bou Noura statten in der Nacht des 24. Tages des Fastenmonates (Ramadan) der relativ gut erhaltenen Moschee ihrer zerstörten Altstadt auf der Hügelkuppe einen Besuch ab.

Wie bei uns im Wallis - heute allerdings nur noch an wenigen Orten - ist auch im Mzab das Gemeinwerk von großer Bedeutung. Alle Männer, von den Betagten, Kranken und Korangelehrten abgesehen, haben zu bestimmten, oft periodisch wiederkehrenden Arbeiten anzutreten, wie Stadtreinigungen, Reparaturarbeiten an öffentlichen Ge- 
bäuden, Gebetsplätzen, Staudämmen, Wasserleitungen usw. Wer am Erscheinen verhindert ist, muß einen Ersatzmann stellen. Die Frontage werden auf dem Marktplatz durch Ausrufen zeitig bekanntgegeben.

Für die Aufrechterhaltung von Ruhe und Ordnung sorgen in den Siedlungen die Nachtwachen. Alle jüngeren Männer kommen in einem bestimmten Kehr zur Erfüllung dieser Aufgabe an die Reihe. In Ben Isguen oder El Ateuf genügen dazu pro Nacht zwei bis vier Mann. Sie haben die Stadttore kurz nach Sonnenuntergang zu schließen und kurz vor Sonnenaufgang wieder zu öffnen. Heimkehrenden, welche am Haupttor klopfen, nach erfolgter Identifizierung Einlaß zu gewähren, durch die Gassen zu zirkulieren und Personen, die sie antreffen - das kommt allerdings selten vor -, anzuhalten und sie nach dem Grunde ihres nächtlichen Herumtreibens zu fragen. Weiter haben sie auch auf Brandausbrüche und Diebe zu achten. Die Wächter sind mit Gewehren bewaffnet.

Von besonderem Interesse ist die Nachtwache von Ghardaia. Sie gliedert sich nach M. Vigourous ${ }^{5}$ in zwei Gruppen: in die der Iduaren oder der gewöhnlichen Nachtwächter, welche die oben aufgezählten Aufgaben zu erfüllen haben, und in die Gruppe der Imessurda. Diese Wächter haben die Pflicht, das Betragen der Bevölkerung zu überwachen, im besonderen festzustellen, ob gewisse Verbote, wie das des Rauchens, Alkoholtrinkens, der Durchführung von Festgelagen, der Prostitution usw., auch tatsächlich eingehalten werden. Sie sind die Sittenrichter der Gemeinschaft. Auf frischer Tat ertappte Sünder werden gestellt und auch sofort und ohne Umstände bestraft. Ihrer wartet je nach der Schwere ihres Vergehens eine mehr oder weniger kräftige Tracht Prügel. In den anderen kleineren Ksur, wo man sich gegenseitig kennt, ist eine solche Überwachungsinstanz nicht notwendig.

Der Nachtwache von Ghardaia gehören 800 bis 900 Mann an. Nur ganz gut beleumdete, verheiratete, sich durch Mut und Stärke auszeichnende jüngere Leute, die auch fähig sind, ein Geheimnis für sich zu behalten, werden in sie aufgenommen. Ihr Dienst erfolgt freiwillig und ist unbezahlt.

Die Nachtwache ist unterteilt in 32 Abteilungen. Jede Nacht haben vier davon, das heißt 60-120 Mann, anzutreten. Darunter hat es Iduaren und Imessurda. Sie streifen sowohl durch die Siedlung als auch durch den Palmgarten, und zwar verschleiert, damit ein aufgespürter Bösewicht sie nicht erkennt und an ihnen Rache nehmen kann.

Die Korporation der Imessurda erinnert an die Knabenschaften Graubündens. Im einen wie im andern Fall handelt es sich um fest geschlossene, straff organisierte Innungen, in die nur ganz unbescholtene Jünglinge - in Graubünden nach ihrer Schulentlassung, in Ghardaia nach ihrer Verheiratung - aufgenommen werden. Ihnen anzugehören, gilt als Ehrensache. Diesen Innungen kommt eine wichtige, in Graubünden heute vielleicht verschwundene, sittenrichterliche Funktion zu, die sich auf die gesamte Bevölkerung - auch in Ghardaia, von den Juden abgesehen - erstreckt. Sie stehen unbedingt im Dienste der Religion und guten Sitte und sind auch politisch von Bedeutung. So hatten nach G. Caduff ${ }^{6}$ die Knabenschaften durch ihr geschlossenes Auftreten bisweilen einen entscheidenden Einfluß auf den Ausgang der Gemeindewahlen. In Ghardaia erlaubte sich die Nachtwache bei schweren Auseinandersetzungen, sowohl im weltlichen (Djemaa) als auch im geistlichen Rat (Halga) zu intervenieren. Sie bildete früher, vor der französischen Besetzung (1853), auch die stehende und allzeit schlagfertige Miliz bei feindlichen Angriffen, zum Beispiel von seiten eines Nomadenstammes. In anderen Gebieten, etwa in Luxemburg, üben die Knabenschaften (nach H. Usener, zitiert von G. Caduff, S. 69) die Aufsicht über die Felder und die reifenden Früchte aus. Das gilt auch für Ghardaia, wo Flurfrevel gleichfalls streng geahndet wird.

5 M. Vigourous: La garde de nuit à Ghardaia. Bulletin de Liaison Saharienne, Alger 1952, Heft 9, S. 9-16.

6 G. Caduff: Die Knabenschaften Graubündens. Chur 1932. 
Den Knabenschaften Graubündens liegen Statuten zugrunde, die dem Uneingeweihten kaum zugänglich sind. Das gleiche gilt auch für die Körperschaft der Imessurda. Beide Institutionen besitzen einen ausgesprochen geheimbündlerischen Charakter, und ihre Mitglieder haben über die internen Angelegenheiten strengstes Stillschweigen zu bewahren. Werden sie fehlbar, kommen sie vor eigenen Disziplinargerichten zur Aburteilung.

Diese Männerbünde unterscheiden sich nur in nebensächlichen Dingen voneinander. Selbst der Umstand, daß den Knabenschaften nur Ledige angehören können, der Nachtwache indessen blo $\beta$ Verheiratete, spielt keine Rolle. Im Mzab, wo jeder Jüngling mit 16 Jahren verheiratet wird, müßte diese Institution, auf der gleichen Voraussetzung wie in Graubünden aufgebaut, hinfällig werden.

Was die Sitten und Bräuche in den beiden Gebieten betrifft, gibt es noch andere merkwürdige Übereinstimmungen. So ist zum Beispiel am einen wie am anderen Ort der Brauch des Bleigießens verbreitet. Während bei uns aber in der Silvesternacht oder am Dreikönigstag Blei gegossen wird, so im Mzab oftmals dann, wenn einen gewisse Dinge, über die man «Klarheit» erlangen möchte, beunruhigen. Will zum Beispiel ein in Algier tätiger Mozabite über die Treue seiner im Mzab zurückgebliebenen Frau Bescheid wissen, greift er nicht selten zu diesem Mittel. Es kam schon vor, daß negative Sinndeutungen der entstandenen Bleifiguren zu Ehescheidungen führten.

Wie soll die auffallende Ähnlichkeit gewisser Sitten und Bräuche zwischen den beiden voneinander so entfernten Gebieten erklärt werden? Ich wage bloß Vermutungen zu äußern. Vielleicht gehen sie in beiden Regionen auf die Römerzeit zurück, vielleicht haben sie sich aber auch da wie dort selbständig entwickelt. Denn gleiche Nöte, wie sie zum Beispiel Trockenzeiten darstellen, lösen ähnliche Reaktionen aus: der Mensch nimmt Zuflucht zum Irrationalen, zu Gott, Gebet und Bittgang.

\section{MGEURS ET COUTUMES AU MZAB}

Certaines mœurs et coutumes connues chez nous en Suisse, surtout dans des régions alpines, sont répandues aussi chez les Mozabites, habitants du Mzab (Sahara algérien), berbères de race et musulmans du rite ibadite. Ainsi p. ex. une bénédiction des palmeraies a lieu chaque printemps. Lors d'unẹ extrême sécheresse, des processions se rendent au bord du lit de l'oued, priant pour que l'eau y afflue. On célèbre aussi des fêtes commémoratives, soit en l'honneur de cheikhs défunts vénérés, anciens chefs des mosquées ibadites, soit pour rappeler des événements historiques.

Comme certaines régions suisses, le $\mathrm{Mzab}$ connaît l'institution des corvées auxquelles tous les hommes aptes au travail ont a participer. Des travaux de réparations aux bâtiments publics, de nettoyage de la ville, l'élévation de digues et le creusement de conduites d'eau et leur entretien se font en corvée. L'institution de la garde de nuit à Ghardaia, capitale du Mzab, est d'un intérêt tout spécial: elle présente une analogie frappante avec certaines organisations secrètes de jeunes gens célibataires dans les Grisons.

\section{ZWEI JAHRE WETTERBEOBACHTUNGEN IN NEPAL (I96I-I963) \\ HANS BOESCH}

Im Jahre 1960 ergab sich in Besprechungen mit dem Schweizerischen Hilfswerk für Außereuropäische Gebiete (SHAG) der Wunsch, in Nepal eine oder mehrere Wetterstationen einzurichten. Das Resultat dieser Besprechungen war, daß a) das Geographische Institut der Universität Zürich die Anschaffung des notwendigen Instrumentariums besorgte und die Auswertung der Resultate vorzunehmen hatte, und daß b) das SHAG den Transport der Instrumente, die Durchführung der Wetterbeobachtungen 